\title{
Compact SOP Representations for Multiple-Output Functions - An Encoding Method using Multiple-Valued Logic -
}

\author{
Tsutomu Sasao \\ Department of Computer Science and Electronics \\ Kyushu Institute of Technology \\ Center for Microelectronic Systems \\ Iizuka 820-8502, Japan
}

\begin{abstract}
This paper shows a method to represent a multipleoutput function: Encoded characteristic function for nonzero outputs $(E C F N)$. The ECFN uses $(n+u)$ binary variables to represent an $n$-input $m$-output function, where $u=\left\lceil\log _{2} m\right\rceil$. The size of the sum-of-products expression$s$ (SOPs) depends on the encoding method of the outputs. For some class of functions, the optimal encoding produces SOPs with $O(n)$ products, while the worst encoding produces SOPs with $O\left(2^{n}\right)$ products. We formulate encoding problem and show a heuristic optimization method. Experimental results using standard benchmark functions show the usefulness of the method.

Index term: Multiple-output function, encoding problem, multiple-valued logic, TDM, SOP, characteristic function.
\end{abstract}

\section{Introduction}

Logic networks usually have many outputs. In most cases, independent representation of each output is inefficient. So, efficient methods to represent multiple-output functions are important. An $n$-input $m$-output combinational logic network is represented by a multiple-output function $F_{1}=\left(f_{0}, f_{1}, \ldots, f_{m-1}\right): B^{n} \rightarrow B^{m}$, where $B=\{0,1\}$.

The first method to represent multiple-output functions is truth tables. Table 1 is a truth table for a 2-input 3-output function. For an $n$-input function, the table requires $2^{n}$ rows, and is too large for large $n$.

The second method is the characteristic function (CF) of the multiple-output function. It is a mapping $F_{2}: B^{n} \times B^{m} \rightarrow B$, where $F_{2}(\vec{a}, \vec{b})=1$ if$\mathrm{f}\left(f_{0}(\vec{a}), f_{1}(\vec{a}), \ldots, f_{m-1}(\vec{a})\right)=\vec{b}$. In this representation, the function requires $m$ auxiliary binary variables $\left\{z_{0}, z_{1}, \ldots, z_{m-1}\right\}$ that represent outputs. $F_{2}$ shows that set of all the valid combinations of the inputs and the outputs. For example, the CF of the multiple-output function shown in Table 1 is

$F_{2}=\bar{x}_{1} \bar{x}_{0} \bar{f}_{0} \bar{f}_{1} \bar{f}_{2} \vee \bar{x}_{1} x_{0} \bar{f}_{0} f_{1} f_{2} \vee x_{1} \bar{x}_{0} \bar{f}_{0} f_{1} f_{2} \vee x_{1} x_{0} f_{0} \bar{f}_{1} f_{2}$.

The CF uses only binary variables, but the size of the representations tends to be very large, since it involves $(n+m)$ binary variables. CFs are used in logic simulation [1], and multi-level logic optimization [6].

The third method is a characteristic function for non-zero outputs (CFN). It is a mapping $F_{3}: B^{n} \times M \rightarrow B$, where $M=\{0,1, \ldots, m-1\}$, and $F_{3}(\vec{a}, j)=1$ iff $f_{j}(\vec{a})=1$, $j \in M$. The CFN has one auxiliary $m$-valued variable $Y$ that represents the output part. For example, the CFN of the multiple-output function shown in Table 1 is

$$
\begin{aligned}
F_{3}= & \bar{x}_{1} x_{0} Y^{1} \vee \bar{x}_{1} x_{0} Y^{2} \vee x_{1} \bar{x}_{0} Y^{1} \vee \\
& x_{1} \bar{x}_{0} Y^{2} \vee x_{1} x_{0} Y^{0} \vee x_{1} x_{0} Y^{2} .
\end{aligned}
$$

A sum-of-products expression (SOP) for a CFN is realized by a programmable logic array (PLA) or an AND-OR twolevel network. SOPs for CFNs are extensively used in logic synthesis [11]. They can be simplified by using multiplevalued logic minimizers such as MINI [7] and ESPRESSOMV [9]. The CFN represents only non-zero input-output combinations, and SOPs for CFNs tend to be more compact than SOPs for CFs.

In this paper, we will consider the fourth method to represent multiple-output function: Encoded characteristic function for non-zero outputs (ECFN). It uses only binary variables and represents a mapping $F_{4}: B^{n} \times B^{u} \rightarrow B$, where $u=\left\lceil\log _{2} m\right\rceil . F_{4}(\vec{a}, \vec{b})=1$ iff $f_{\nu(\vec{b})}(\vec{a})=1$, where $\nu(\vec{b})$ denotes the integer represented by the binary vector $\vec{b}$. For example, the ECFN of the multiple-output function shown in Table 1 is

$$
\begin{aligned}
F_{4}= & \bar{x}_{1} x_{0} \bar{z}_{1} z_{0} \vee \bar{x}_{1} x_{0} z_{1} \bar{z}_{0} \vee x_{1} \bar{x}_{0} \bar{z}_{1} z_{0} \vee \\
& x_{1} \bar{x}_{0} z_{1} \bar{z}_{0} \vee x_{1} x_{0} \bar{z}_{1} \bar{z}_{0} \vee x_{1} x_{0} z_{1} \bar{z}_{0}
\end{aligned}
$$

Note that $F_{4}$ is also represented as

$$
F_{4}=\bar{z}_{1} \bar{z}_{0} f_{0} \vee \bar{z}_{1} z_{0} f_{1} \vee z_{1} \bar{z}_{0} f_{2} \vee z_{1} z_{0} f_{3} .
$$

ECFNs are useful for FPGA design [8], logic emulation [2], and logic simulation [12]. 
Table 1. Truth table for a 2-input 3-output function.

\begin{tabular}{cc|ccc}
\hline$x_{1}$ & $x_{0}$ & $f_{0}$ & $f_{1}$ & $f_{2}$ \\
\hline 0 & 0 & 0 & 0 & 0 \\
0 & 1 & 0 & 1 & 1 \\
1 & 0 & 0 & 1 & 1 \\
1 & 1 & 1 & 0 & 1 \\
\hline
\end{tabular}

\section{ECFN and Encoding Problem}

In this section, we will define encoded characteristic functions for non-zero outputs (ECFN's) and formulate their encoding program.

Definition $2.1 x^{0}=\bar{x}$, and $x^{1}=x$.

Definition 2.2 For an m-output function $f_{i}(i=$ $0,1, \ldots, m-1)$, an encoded characteristic function for nonzero outputs (ECFN) is defined as

$$
F=\bigvee_{i=0}^{m-1} z_{u-1}^{b_{u-1}} z_{u-2}^{b_{u-2}} \cdots z_{0}^{b_{0}} f_{i}
$$

where $\vec{b}=\left(b_{u-1}, b_{u-2}, \ldots, b_{0}\right)$ is a binary representation of an integer $i$, and $u=\left\lceil\log _{2} m\right\rceil$.

Note that $z_{0}, z_{1}, \ldots$, and $z_{u-1}$ are auxiliary variables that represent outputs. In the above definition, the integer $i$ is encoded by a binary vector $\vec{b}$ in a natural way. However, by changing the encoding, we can often simplify the representation.

Example 2.1 Consider a four-output function $F=$ $\left(f_{0}, f_{1}, f_{2}, f_{3}\right)$. Encoding 1 in Table 2 produces the ECFN

$$
F_{1}=\bar{z}_{1} \bar{z}_{0} f_{0} \vee \bar{z}_{1} z_{0} f_{1} \vee z_{1} \bar{z}_{0} f_{2} \vee z_{1} z_{0} f_{3} .
$$

Consider the case where $f_{0}=0, f_{1}=x_{0}, f_{2}=x_{1} \vee x_{0}$, and $f_{3}=x_{1}$. Then, we have

$$
\begin{aligned}
F_{1} & =\bar{z}_{1} \bar{z}_{0} 0 \vee \bar{z}_{1} z_{0} x_{0} \vee z_{1} \bar{z}_{0}\left(x_{1} \vee x_{0}\right) \vee z_{1} z_{0} x_{1} \\
& =\bar{z}_{1} z_{0} x_{0} \vee z_{1} \bar{z}_{0} x_{0} \vee z_{1} \bar{z}_{0} x_{1} \vee z_{1} z_{0} x_{1} \\
& =\left(\bar{z}_{1} z_{0} \vee z_{1} \bar{z}_{0}\right) x_{0} \vee\left(z_{1} \bar{z}_{0} \vee z_{1} z_{0}\right) x_{1} \\
& =\bar{z}_{1} z_{0} x_{0} \vee z_{1} \bar{z}_{0} x_{0} \vee z_{1} x_{1} .
\end{aligned}
$$

However, Encoding 2 in Table 2 produces the ECFN

$$
F_{2}=\bar{z}_{1} \bar{z}_{0} f_{0} \vee \bar{z}_{1} z_{0} f_{1} \vee z_{1} \bar{z}_{0} f_{3} \vee z_{1} z_{0} f_{2} .
$$

In this case, we have

$$
\begin{aligned}
F_{2} & =\bar{z}_{1} \bar{z}_{0} 0 \vee \bar{z}_{1} z_{0} x_{0} \vee z_{1} \bar{z}_{0} x_{1} \vee z_{1} z_{0}\left(x_{1} \vee x_{0}\right) \\
& =\bar{z}_{1} z_{0} x_{0} \vee z_{1} \bar{z}_{0} x_{1} \vee z_{1} z_{0} x_{0} \vee z_{1} z_{0} x_{1} \\
& =z_{0} x_{0} \vee z_{1} x_{1} .
\end{aligned}
$$

Note that Encoding 1 requires three products, while Encoding 2 requires only two products.

(End of Example)
Table 2. Encoding methods for four-output function.

\begin{tabular}{cc|c|c|c}
\hline$z_{1}$ & $z_{0}$ & Encoding 1 & Encoding 2 & Encoding 3 \\
\hline 0 & 0 & $f_{0}$ & $f_{0}$ & $f_{0}$ \\
0 & 1 & $f_{1}$ & $f_{1}$ & $f_{3}$ \\
1 & 0 & $f_{2}$ & $f_{3}$ & $f_{2}$ \\
1 & 1 & $f_{3}$ & $f_{2}$ & $f_{1}$ \\
\hline
\end{tabular}

Example 2.2 Consider an 8-output function $\left(f_{0}, f_{1}, \ldots\right.$, $f_{7}$ ), where $f_{0}=0, f_{1}=x_{0}, f_{2}=x_{1}, f_{3}=x_{2}, f_{4}=x_{0} \vee x_{1}$, $f_{5}=x_{0} \vee x_{2}, f_{6}=x_{1} \vee x_{2}, f_{7}=x_{0} \vee x_{1} \vee x_{2}$.

In this case, we need three auxiliary variables $z_{0}, z_{1}$, and $z_{2}$ to represent 8 outputs. Encoding 1 in Table 3 produces the ECFN

$$
\begin{aligned}
F_{1}= & \bar{z}_{2} \bar{z}_{1} \bar{z}_{0} f_{0} \vee \bar{z}_{2} \bar{z}_{1} z_{0} f_{1} \vee \bar{z}_{2} z_{1} \bar{z}_{0} f_{2} \vee \bar{z}_{2} z_{1} z_{0} f_{3} \vee \\
& z_{2} \bar{z}_{1} \bar{z}_{0} f_{4} \vee z_{2} \bar{z}_{1} z_{0} f_{5} \vee z_{2} z_{1} \bar{z}_{0} f_{6} \vee z_{2} z_{1} z_{0} f_{7} .
\end{aligned}
$$

Thus, we have

$$
\begin{aligned}
F_{1}= & \bar{z}_{2} \bar{z}_{1} \bar{z}_{0} 0 \vee \bar{z}_{2} \bar{z}_{1} z_{0} x_{0} \vee \bar{z}_{2} z_{1} \bar{z}_{0} x_{1} \vee \bar{z}_{2} z_{1} z_{0} x_{2} \vee \\
& z_{2} \bar{z}_{1} \bar{z}_{0}\left(x_{0} \vee x_{1}\right) \vee z_{2} \bar{z}_{1} z_{0}\left(x_{0} \vee x_{2}\right) \vee \\
& z_{2} z_{1} \bar{z}_{0}\left(x_{1} \vee x_{2}\right) \vee z_{2} z_{1} z_{0}\left(x_{0} \vee x_{1} \vee x_{2}\right) \\
= & \bar{z}_{1} z_{0} x_{0} \vee z_{1} \bar{z}_{0} x_{1} \vee z_{1} z_{0} x_{2} \vee z_{2} \bar{z}_{1} x_{0} \vee z_{2} \bar{z}_{0} x_{1} \vee \\
& z_{2} z_{0} x_{2} \vee z_{2} z_{1} x_{2} \vee z_{2} z_{0} x_{0} \vee z_{2} z_{1} x_{1} .
\end{aligned}
$$

However, Encoding 2 in Table 2 produces the ECFN

$$
\begin{aligned}
F_{2}= & \bar{z}_{2} \bar{z}_{1} \bar{z}_{0} f_{0} \vee \bar{z}_{2} \bar{z}_{1} z_{0} f_{1} \vee \bar{z}_{2} z_{1} \bar{z}_{0} f_{2} \vee \bar{z}_{2} z_{1} z_{0} f_{4} \vee \\
& z_{2} \bar{z}_{1} \bar{z}_{0} f_{3} \vee z_{2} \bar{z}_{1} z_{0} f_{5} \vee z_{2} z_{1} \bar{z}_{0} f_{6} \vee z_{2} z_{1} z_{0} f_{7}
\end{aligned}
$$

Thus, we have

$$
\begin{aligned}
F_{2}= & \bar{z}_{2} \bar{z}_{1} \bar{z}_{0} 0 \vee \bar{z}_{2} \bar{z}_{1} z_{0} x_{0} \vee \bar{z}_{2} z_{1} \bar{z}_{0} x_{1} \vee \\
& \bar{z}_{2} z_{1} z_{0}\left(x_{0} \vee x_{1}\right) \vee z_{2} \bar{z}_{1} \bar{z}_{0} x_{2} \vee z_{2} \bar{z}_{1} z_{0}\left(x_{0} \vee x_{2}\right) \vee \\
& z_{2} z_{1} \bar{z}_{0}\left(x_{1} \vee x_{2}\right) \vee z_{2} z_{1} z_{0}\left(x_{0} \vee x_{1} \vee x_{2}\right) \\
= & \left(\bar{z}_{2} \bar{z}_{1} z_{0} \vee \bar{z}_{2} z_{1} z_{0} \vee z_{2} \bar{z}_{1} z_{0} \vee z_{2} z_{1} z_{0}\right) x_{0} \vee \\
& \left(\bar{z}_{2} z_{1} \bar{z}_{0} \vee \bar{z}_{2} z_{1} z_{0} \vee z_{2} z_{1} \bar{z}_{0} \vee z_{2} z_{1} z_{0}\right) x_{1} \vee \\
& \left(z_{2} \bar{z}_{1} \bar{z}_{0} \vee z_{2} \bar{z}_{1} z_{0} \vee z_{2} z_{1} \bar{z}_{0} \vee z_{2} z_{1} z_{0}\right) x_{2} \\
= & z_{0} x_{0} \vee z_{1} x_{1} \vee z_{2} x_{2} .
\end{aligned}
$$

Note that Encoding 1 requires 9 products, while Encoding 2 requires only three products.

(End of Example)

As shown in the previous examples, good encodings produce simpler SOPs.

For an $m$-output function, we need $u=\left\lceil\log _{2} m\right\rceil$ auxiliary variables $\left\{z_{0}, z_{1}, \ldots, z_{u-1}\right\}$ to represent $m$ outputs. So, the number of different encodings is

$$
\frac{2^{u} !}{\left(2^{u}-m\right) !} \text {. }
$$


Table 3. Encoding methods for 8-output function.

\begin{tabular}{ccc|c|c}
\hline$z_{2}$ & $z_{1}$ & $z_{0}$ & Encoding 1 & Encoding 2 \\
\hline 0 & 0 & 0 & $f_{0}$ & $f_{0}$ \\
0 & 0 & 1 & $f_{1}$ & $f_{1}$ \\
0 & 1 & 0 & $f_{2}$ & $f_{2}$ \\
0 & 1 & 1 & $f_{3}$ & $f_{4}$ \\
\hline 1 & 0 & 0 & $f_{4}$ & $f_{3}$ \\
1 & 0 & 1 & $f_{5}$ & $f_{5}$ \\
1 & 1 & 0 & $f_{6}$ & $f_{6}$ \\
1 & 1 & 1 & $f_{7}$ & $f_{7}$ \\
\hline
\end{tabular}

However, the number of products in the SOP is invariant under the complement and/or permutation of the auxiliary variables. Thus, to find the encoding for an ECFN that requires the least number of products in an SOP, we have only to consider

$$
N=\frac{2^{u} !}{\left(2^{u}-m\right) ! 2^{u} u !}=\frac{\left(2^{u}-1\right) !}{\left(2^{u}-m\right) ! u !}
$$

different encodings. For $m=4$, we have $u=2$, and we need only to consider $N=\frac{3 !}{1 ! 2 !}=3$ different encodings. Note that Encoding 3 in Table 2 also requires three products to represent the function in Example 2.1.

Given a CFN $F: B^{n} \times M \rightarrow B$, where $M=$ $\{0,1, \ldots, m-1\}$, we can formulate

Problem 2.1 (Encoding problem for an ECFN) Given a function $B^{n} \times M \rightarrow B$, where $M=\{0,1,2, \ldots, m-1\}$. Represent the $m$-valued variable by using $u=\left\lceil\log _{2} m\right\rceil$ binary variables so that the resulting SOP has the least number of products.

This problem is similar to

Problem 2.2 (Input encoding problem) Given a function $B^{n} \times M \rightarrow B$, where $M=\{0,1,2, \ldots, m-1\}$. Represent the $m$-valued variable by using the sufficient number of auxiliary binary variables, so that the resulting SOP has the least number of products.

Note that in Problem 2.1, the number of the auxiliary variables is fixed to $u=\left\lceil\log _{2} m\right\rceil$, while in Problem 2.2, the number of auxiliary variables can be more than $u$. Both of these problems are intractable and require heuristic algorithms to solve them. Several algorithms have been developed for Problem 2.2 [5, 13, 14]. However, to our knowledge, Problem 2.1 is formulated for the first time, and no good algorithm is known.

\section{Encoding Algorithm for ECFN}

In this section, we will consider a heuristic algorithm to encode an $m$-valued variables by using $u=\left\lceil\log _{2} m\right\rceil$ binary

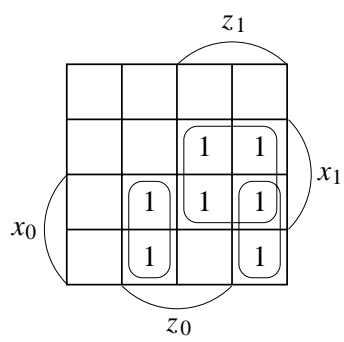

(a) Encoding 1.

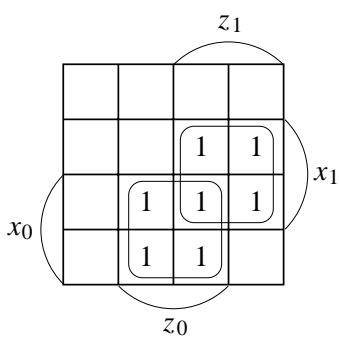

(b) Encoding 2 .
Figure 1. SOPs for ECFNs with different encodings.

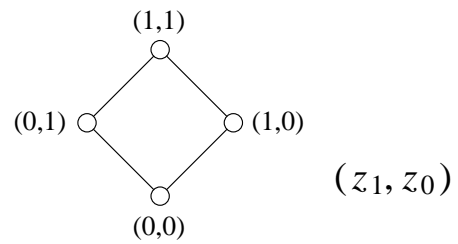

Figure 2. 2-dimensional cube.

variables, so that the resulting SOP has the least number of products. To show the idea of the heuristic, we will use

Example 3.3 Consider the 4-output function $f_{0}=0, f_{1}=$ $x_{0}, f_{2}=x_{0} \vee x_{1}, f_{3}=x_{1}$. The positional cubes $[7,11,6]$ for the ECFN are

\begin{tabular}{cccccccc}
\hline$x_{1}$ & & $x_{0}$ & & $f_{0}$ & $f_{1}$ & $f_{2}$ & $f_{3}$ \\
\hline 11 & - & 01 & - & 0 & 1 & 0 & 0 \\
11 & - & 01 & - & 0 & 0 & 1 & 0 \\
01 & - & 11 & - & 0 & 0 & 1 & 0 \\
01 & - & 11 & - & 0 & 0 & 0 & 1 \\
\hline
\end{tabular}

They are simplified to

\begin{tabular}{cccccccc}
\hline$x_{1}$ & & $x_{0}$ & & $f_{0}$ & $f_{1}$ & $f_{2}$ & $f_{3}$ \\
\hline 11 & - & 01 & - & 0 & 1 & 1 & 0 \\
01 & - & 11 & - & 0 & 0 & 1 & 1 \\
\hline
\end{tabular}

Encodings 1 and 2 produce maps in Fig. $1(a)$ and (b), respectively. Note that Encoding 1 requires three products, while Encoding 2 requires only two products.

In general, an encoding for an ECFN corresponds to assign m output functions to the nodes of the u-dimensional cube. In this example, $m=4$ and $u=2$. Thus, we have to assign four functions $f_{0}, f_{1}, f_{2}, f_{3}$ to four nodes of the 2-dimensional cube in Fig. 2. 
We can find a good assignment from the output part of the minimized positional cubes:

\begin{tabular}{cccc}
\hline$f_{0}$ & $f_{1}$ & $f_{2}$ & $f_{3}$ \\
\hline 0 & 1 & 1 & 0 \\
0 & 0 & 1 & 1 \\
\hline
\end{tabular}

This is called a constraint matrix [6]. The first row of the constraint matrix shows that $f_{1}$ and $f_{2}$ must be adjacent to be represented by a single product. And the second row of the constraint matrix shows that $f_{2}$ and $f_{3}$ must be adjacent to be represented by a single product. Encoding 1 in Table 2 (Fig. 3(a)) does not satisfy the conditions, while Encoding 2 (Fig. 3(b)) satisfies the conditions. So, we can expect that Encoding 2 produces simpler SOP than Encoding 1.

(End of Example)

As shown in Example 3.3, the constraint matrix is useful to find a good encoding. To find a good encoding, we will use the Merit matrix. The value of $\operatorname{Merit}(i, j)$, where $i, j \in M$ and $M=\{0,1,2, \ldots, m-1\}$ is large when $f_{i}$ and $f_{j}$ should be assigned to the adjacent nodes in the $u$-dimensional cube.

\section{Algorithm 3.1 (Derivation of the Merit Matrix)}

1. From the minimized SOP of the CFN, obtain the constraint matrix. Ignore the rows with all 1's. Ignore the rows with single 1's.

2. Let $\operatorname{Merit}(j, k) \leftarrow 0.0$, where $j, k \in M$ and $M=$ $\{0,1, \ldots, m-1\}$.

3. For each row $i$ in the constraint matrix, let $S_{i}$ be the set of indexes of columns that have l's in the row $i$. Let $\left|S_{i}\right|$ be the number of elements in $S_{i}$. For each pair $(j, k) \in S_{i}$, do Merit $(j, k) \leftarrow \operatorname{Merit}(j, k)+\frac{1}{\left|S_{i}\right|-1}$.

4. If $\operatorname{Merit}(j, k)=0.0$ and $j, k \leq m$ and $j \neq k$, then let $\operatorname{Merit}(j, k) \leftarrow-1+\frac{u}{2^{n}}$.

Example 3.4 Consider the four-output function in Example 3.3, where $m=4$. By using Algorithm 3.1, we will obtain the merit matrix.

1) $S_{1}=\{1,2\}, S_{2}=\{2,3\}$, and $\left|S_{1}\right|=\left|S_{2}\right|=2$.

2) For $i=1$, we have

$$
\operatorname{Merit}(1,2) \leftarrow 1.0, \operatorname{Merit}(2,1) \leftarrow 1.0 .
$$

For $i=2$, we have

$$
\operatorname{Merit}(2,3) \leftarrow 1.0, \operatorname{Merit}(3,2) \leftarrow 1.0 .
$$

3) In Step 3, we have

$$
\text { Merit }=\begin{aligned}
& 0 \\
& 1 \\
& 2 \\
& 3
\end{aligned}\left[\begin{array}{cccc}
0 & 1 & 2 & 3 \\
0.0 & 0.0 & 0.0 & 0.0 \\
0.0 & 0.0 & 1.0 & 0.0 \\
0.0 & 1.0 & 0.0 & 1.0 \\
0.0 & 0.0 & 1.0 & 0.0
\end{array}\right] .
$$

4) Since $n=2$ and $u=2$ in Step 4, we have

$$
-1.0+\frac{u}{2^{n}}=-1.0+0.5=-0.5 \text {. }
$$

Thus, we have

$$
\text { Merit }=\begin{aligned}
& 0 \\
& 1 \\
& 2 \\
& 3
\end{aligned}\left[\begin{array}{rrrr}
0 & 1 & 2 & 3 \\
0.0 & -0.5 & -0.5 & -0.5 \\
-0.5 & 0.0 & 1.0 & -0.5 \\
-0.5 & 1.0 & 0.0 & 1.0 \\
-0.5 & -0.5 & 1.0 & 0.0
\end{array}\right] .
$$

\section{Algorithm 3.2 (Encoding of an ECFN)}

1. As an initial solution, assign functions $f_{0}, f_{1}, \ldots, f_{m-1}$ to distinct nodes of the u-dimensional cube. Let $f_{0}$ be assigned to the node $(0,0, \ldots, 0)$. When $m<2^{u}$, assign dummy functions to the remaining nodes.

2. Gain $\leftarrow \sum \operatorname{Merit}(j, k)$, where the sum is obtained for the adjacent nodes $(j, k)$ in the u-dimensional cube.

3. Fix the function $f_{0}$ to the node $(0,0, \ldots, 0)$. For other $2^{u}-1$ functions, choose a pair of functions. If Gain increases by the exchange of the functions in the pair, then exchange the functions. Otherwise, do not exchange the functions. Repeat this operation while Gain increases.

4. Fix the function $f_{0}$ to the node $(0,0, \ldots, 0)$. For other $2^{u}-1$ functions, choose a pair of functions. If Gain do not decrease by the exchange of the functions in the pair, then exchange the functions. Otherwise, do not exchange the functions. Repeat this operation while Gain increases.

5. Do the same thing as Step 3.

6. If Gain increased in Step 5, then go to Step 3. Otherwise stop.

Example 3.5 By using Algorithm 3.2, we will obtain a good encoding for the function in Example 3.3.

1) The initial assignment is given in Fig. 3(a).

2) $\operatorname{Gain}=\operatorname{Merit}(0,1)+\operatorname{Merit}(0,2)+\operatorname{Merit}(1,3)+$ $\operatorname{Merit}(2,3)=-0.5-0.5-0.5+1.0=-0.5$.

3) Choose the pair $\left(f_{2}, f_{3}\right)$, and interchange the functions. Then, we have the assignment in Fig. 3(b).

4) In this case, Gain $=\operatorname{Merit}(0,1)+\operatorname{Merit}(0,3)+$ Merit $(1,2)+M \operatorname{erit}(2,3)=-0.5-0.5+1.0+1.0=$ 1.0. Since, Gain has increased, we keep this exchange.

5) Choose the pair $\left(f_{1}, f_{3}\right)$ in Fig. 3(a), and interchange the functions. Then, we have the assignment in Fig. 3(c). 


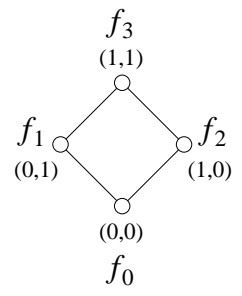

(a)

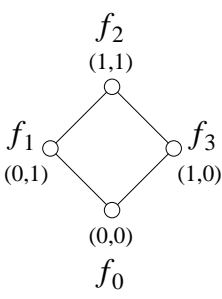

(b)

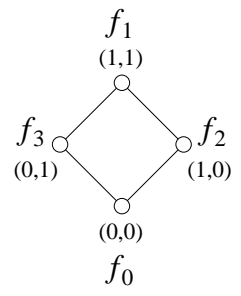

(c)

\section{Figure 3. Assignments of four outputs to 2- dimensional cubes.}

6) In this case, Gain $=\operatorname{Merit}(0,2)+\operatorname{Merit}(0,3)+$ Merit $(1,2)+$ Merit $(1,3)=-0.5-0.5+1.0-0.5=$ -0.5 , which is the same as the case for Fig. 3(a). It is clear that Figs. 3(b) gives the best encoding.

(End of Example)

Note that Algorithm 3.2 does not always produces the optimal solution.

Theorem 3.1 The n-input $2^{n}$-output function $f_{i}(\vec{x})=$ $\bigvee_{j=0}^{n-1} a_{i j} x_{j}\left(i=0,1, \ldots, 2^{n}-1\right)$, is represented by an SOP for an ECFN with $n$ products, where $\vec{x}=$ $\left(x_{n-1}, x_{n-2}, \ldots, x_{0}\right)$ and $\vec{a}_{i}=\left(a_{i n-1}, a_{i n-2}, \ldots, a_{i 0}\right)$ is a binary representation of the integer $i$.

Example 3.6 When $n=3$, we have $f_{0}=0, f_{1}=x_{0}$, $f_{2}=x_{1}, f_{3}=x_{1} \vee x_{0}, f_{4}=x_{2}, f_{5}=x_{2} \vee x_{0}, f_{6}=x_{2} \vee x_{1}$, $f_{7}=x_{2} \vee x_{1} \vee x_{0}$. In this case the ECFN is represented by

$$
\begin{aligned}
F= & \bar{z}_{2} \bar{z}_{1} \bar{z}_{0} f_{0} \vee \bar{z}_{2} \bar{z}_{1} z_{0} f_{1} \vee \bar{z}_{2} z_{1} \bar{z}_{0} f_{2} \vee \bar{z}_{2} z_{1} z_{0} f_{3} \vee \\
& z_{2} \bar{z}_{1} \bar{z}_{0} f_{4} \vee z_{2} \bar{z}_{1} z_{0} f_{5} \vee z_{2} z_{1} \bar{z}_{0} f_{6} \vee z_{2} z_{1} z_{0} f_{7} \\
= & z_{0} x_{0} \vee z_{1} x_{1} \vee z_{2} x_{2} \\
= & \bigvee_{i=0}^{2} z_{i} x_{i} .
\end{aligned}
$$

(End of Example)

Theorem 3.2 The n-input $2^{n}$-output function $f_{i}(\vec{x})=$ $\bigvee_{j=0}^{n-1} x_{j}^{\bar{a}_{i j}}\left(i=0,1,2, \ldots, 2^{n}-1\right)$, is represented by an SOP for an ECFN with $2 n$ products, where $\vec{x}=$ $\left(x_{n-1}, x_{n-2}, \ldots, x_{0}\right)$ and $\vec{a}_{i}=\left(a_{i n-1}, a_{i n-2}, \ldots, a_{i 0}\right)$ is a binary representation of the integer $i$.

Example 3.7 When $n=3$, we have

$$
\begin{aligned}
& f_{0}=x_{2} \vee x_{1} \vee x_{0}, \\
& f_{1}=x_{2} \vee x_{1} \vee \bar{x}_{0}, \\
& f_{2}=x_{2} \vee \bar{x}_{1} \vee x_{0}, \\
& f_{3}=x_{2} \vee \bar{x}_{1} \vee \bar{x}_{0},
\end{aligned}
$$

$$
\begin{aligned}
& f_{4}=\bar{x}_{2} \vee x_{1} \vee x_{0}, \\
& f_{5}=\bar{x}_{2} \vee x_{1} \vee \bar{x}_{0}, \\
& f_{6}=\bar{x}_{2} \vee \bar{x}_{1} \vee x_{0}, \\
& f_{7}=\bar{x}_{2} \vee \bar{x}_{1} \vee \bar{x}_{0} .
\end{aligned}
$$

In this case, the ECFN is represented by

$$
\begin{aligned}
F= & \bar{z}_{2} \bar{z}_{1} \bar{z}_{0} f_{0} \vee \bar{z}_{2} \bar{z}_{1} z_{0} f_{1} \vee \bar{z}_{2} z_{1} \bar{z}_{0} f_{2} \vee \bar{z}_{2} z_{1} z_{0} f_{3} \vee \\
& z_{2} \bar{z}_{1} \bar{z}_{0} f_{4} \vee z_{2} \bar{z}_{1} z_{0} f_{5} \vee z_{2} z_{1} \bar{z}_{0} f_{6} \vee z_{2} z_{1} z_{0} f_{7} \\
= & \left(z_{0} \oplus x_{0}\right) \vee\left(z_{1} \oplus x_{1}\right) \vee\left(z_{2} \oplus x_{2}\right) \\
= & \bigvee_{i=0}^{2}\left(z_{i} \oplus x_{i}\right) .
\end{aligned}
$$

(End of Example)

Definition 3.3 Let $\tau(M S O P, C F N)$ denote the number of products in a minimum sum-of-products expression (MSOP) for characteristic function for non-zero outputs (CFN), i.e., the number of products for minimized PLA for the multipleoutput function. $\tau(M S O P, E C F N)$ and $\tau(M S O P, C F)$ are defined similarly.

Theorem 3.3 $\tau(M S O P, C F N) \leq \tau(M S O P, E C F N)$.

Definition 3.4 Let $\tau(M S O P, C F N(n))$ denote the number of products in a MSOP for CFN of an n-variable function. $\tau(M S O P, C F(n))$ is defined similarly.

Theorem 3.4 $\tau(M S O P, C F N(n)) \leq 2^{n}, \quad \tau(M S O P$, $C F(n)) \leq 2^{n}$.

\section{Experimental Results}

We developed programs for Algorithms 3.1 and 3.2, and minimized SOPs for various benchmark functions.

Table 4 shows the experimental results, where Name denotes the function name; In denotes the number of input variables; Out denotes the number of output variables; $C F N$ denotes the number of products in the SOP for CFN (i.e., the number of products in the minimized PLA); ECFN denotes the number of products in the SOP for ECFN; Good denotes the ECFN obtained by Algorithm 3.2; $\mathrm{Org}$ denotes the ECFN obtained by the straightforward encoding; Bad denotes the ECFN obtained by Algorithm 3.2 modified so that the Gain becomes small as possible; $C F$ denotes the number of products in the SOP for CF. Table 4 shows that we can reduce the number of products by considering the encoding. Also, we can verify that Theorem 3.3 holds. In many cases, we could not obtain SOPs for CF due to memory overflow.

Table 5 shows the numbers of products in the SOPs for ECFNs for the multiple-output functions defined in Theorem 3.1. This table shows that the number of products in Bad encoding increases exponentially as $n$ increases. Thus, we have 
Table 4. Number of Products in SOPs.

\begin{tabular}{|l|r|r|r|r|r|r|r|}
\hline & & & CFN & \multicolumn{2}{|c|}{ ECFN } & CF \\
\cline { 4 - 7 } Name & In & Out & PLA & Good & Org & Bad & \\
\hline 5xp1 & 7 & 10 & 63 & 67 & 69 & 74 & 128 \\
amd & 14 & 24 & 66 & 108 & 125 & 158 & 137 \\
apex1 & 45 & 45 & 206 & 522 & 719 & 898 & \\
apex3 & 54 & 50 & 280 & 475 & 496 & 627 & \\
apex4 & 9 & 19 & 429 & 772 & 833 & 983 & 438 \\
b12 & 15 & 9 & 44 & 46 & 48 & 53 & 208 \\
clip & 9 & 5 & 118 & 126 & 132 & 141 & 430 \\
duke2 & 22 & 29 & 86 & 138 & 175 & 198 & \\
in4 & 32 & 20 & 211 & 255 & 305 & 350 & \\
misex1 & 8 & 7 & 12 & 18 & 23 & 26 & 18 \\
misex2 & 25 & 28 & 28 & 28 & 29 & 29 & 472 \\
misex3 & 14 & 14 & 690 & 904 & 962 & 1121 & 1938 \\
misg & 56 & 23 & 69 & 70 & 73 & 75 & \\
mish & 94 & 43 & 82 & 84 & 89 & 91 & \\
misj & 35 & 14 & 35 & 35 & 48 & 48 & \\
mlp4 & 8 & 8 & 126 & 130 & 133 & 144 & 227 \\
opa & 17 & 69 & 80 & 194 & 244 & 298 & \\
risc & 8 & 31 & 27 & 33 & 43 & 46 & 29 \\
seq & 41 & 35 & 350 & 730 & 1100 & 1398 & \\
soar & 83 & 94 & 357 & 378 & 448 & 482 & \\
ti & 47 & 72 & 214 & 335 & 388 & 511 & \\
x2dn & 82 & 56 & 104 & 106 & 114 & 116 & \\
\hline
\end{tabular}

Table 5. Number of Products in SOPs for ECFNs in Theorem 3.1.

\begin{tabular}{|c|c|r|}
\hline$n$ & Good & Bad \\
\hline 3 & 3 & 8 \\
4 & 4 & 20 \\
5 & 5 & 48 \\
6 & 6 & 112 \\
7 & 7 & 274 \\
8 & 8 & 633 \\
\hline
\end{tabular}

Conjecture 4.1 An n-input $2^{n}$-output function exists that requires $O(n)$ and $O\left(2^{n}\right)$ products in SOPs for the ECFN with the encoding optimized and an ECFN with the encoding un-optimized, respectively.

\section{Conclusion and Comments}

In this paper, we presented a new method to represent a multiple-output function: An encoding characteristic function for non-zero outputs (ECFN). An ECFN uses only binary variables, and its SOP can be simplified by considering the encoding. We formulated the encoding problem, and presented a heuristic optimization method. We also showed an $n$-input $2^{n}$-output function that requires $O(n)$ products in an MSOP for one encoding, and $O\left(2^{n}\right)$ products for other encoding.

In this paper, we only considered the encoding for SOPs. However, the more interesting problem is the encoding for
BDDs, which is useful for logic simulator [12].

\section{Acknowledgments}

This work was supported in part by a Grant in Aid for Scientific Research of the Ministry of Education, Culture, Sports, Science and Technology of Japan. Mr. M. Matsuura prepared the $\mathrm{IAT}_{\mathrm{E}} \mathrm{X}$ files.

\section{References}

[1] P. Asher and S. Malik, "Fast functional simulation using branching programs," ICCAD, pp. 408-412, Oct. 1995.

[2] J. Babb, R. Tessier, M. Dahl, S. Z. Hanono, D. M. Hoki, and A. Agarwal, "Logic emulation with virtual wires," IEEE Trans on CAD, Vol. 16, No. 6, pp. 609-626, June 1997.

[3] R. E. Bryant, "Graph-based algorithms for Boolean function manipulation," IEEE Trans. Comput., Vol. C-35, No. 8, pp. 677-691(Aug. 1986).

[4] E. Cerny and M. A. Martin, "An approach to unified methodology of combinational switching circuits," IEEE TC, Vol. C26, No. 8, pp. 745-756, Aug. 1977.

[5] G. De Micheli, R. K. Brayton, and A. L. SangiovanniVincentelli, "Optimal state assignment of finite state machine," IEEE Trans. on CAD, vol. CAD-4, No. 3, pp. 262285, July 1985.

[6] G. De Micheli, Synthesis and Optimization of Digital Circuits, McGraw-Hill, 1994.

[7] S. J. Hong, R. G. Cain, and D. L. Ostapko, "MINI: A heuristic approach for logic minimization," IBM J. Res. and Develop., pp. 443-458, Sept. 1974.

[8] J.-H. R. Jian, J.-Y. Jou, and J.-D. Huang, "Compatible class encoding in hyper-function decomposition for FPGA synthesis," Design Automation Conference, pp. 712-717, June 1998.

[9] R. L. Rudell and A. L. Sangiovanni-Vincentelli, "Multiplevalued minimization for PLA optimization," IEEE TCAD, Vol. CAD-6, No. 5, pp. 727-750, Sept. 1987.

[10] T. Sasao, "An application of multiple-valued logic to a design of programmable logic arrays," ISMVL-78, pp. 65-72, May 1978.

[11] T. Sasao, Switching Theory for Logic Synthesis, Kluwer Academic Publishers, 1999.

[12] T. Sasao, M. Matsuura, and Y. Iguchi, "Cascade realization of multiple-output function and its application to reconfigurable hardware," (draft).

[13] T. Villa and A. Sangiovanni-Vincentelli, "NOVA: State assignment for finite state machines for optimal two-level logic implementation," IEEE TCAD, Vol. CAD-9, No. 9, pp. 905924, Sept. 1990.

[14] S. Yang and M. Ciesielski, "Optimum and suboptimum algorithm for input encoding and its relationship to logic minimization," IEEE Trans. CAD, Vol. CAD-10, No. 1 pp. 4-12, Jan. 1991. 